\title{
BIBLIOGRAPHY
}

1. C. Chevalley and A. Weil, Über das Verhalten der Integrale. 1. Gattung bei Automorphismen des Funktionenkörpers, Abh. Math. Sem. Univ. Hamburg 10 (1934), 358-361.

2. R. C. Gunning, Lectures on modular forms, Princeton Univ. Press, Princeton, N. J., 1962.

3. J. Lewittes, Automorphisms of compact Riemann surfaces, Thesis, Yeshiva University, New York, 1962.

4. B. Schoeneberg, Über die Weierstrass Punkte in den Körpern der Elliptischen Modulfunktionen, Abh. Math. Sem. Univ. Hamburg 17 (1951), 104-111.

Yeshiva UNIVERSITY

\section{SOME RESULTS ON THE EXTENSION OF OPERATORS ${ }^{1}$}

\section{BY JORAM LINDENSTRAUSS}

Communicated by Felix Browder, April 15, 1963

1. Introduction. The subject of the present note is closely related to questions treated in [6] and [7] (cf. also [8]). In $\$ 2$ we state some results showing that certain extension properties for operators with a two-dimensional range imply extension properties for a much larger class of operators. Extension properties for operators with a twodimensional range are, in a sense, the weakest possible, since by the Hahn Banach theorem operators with a one-dimensional range can always be extended in a norm preserving manner.

The results stated in $\$ 3$ demonstrate the rôle of finite dimensional spaces whose unit cell is a polyhedron in some problems concerning norm preserving extension of operators. Proofs of the results stated here will be published elsewhere.

I wish to express my thanks to Professor S. Kakutani for many valuable discussions concerning the subject of this note.

Notations. All Banach spaces are assumed to be over the reals. $S_{X}$ denotes the unit cell $\{x ;\|x\| \leqq 1\}$ of the Banach space $X$. By "cell" we mean a translate of $r S_{X}, r>0$. All operators are assumed to be linear and bounded.

2. Our first theorem complements the main result of [7] (cf. also $[8$, Theorem 1]).

THEOREM 1. Let $X$ be a Banach space such that $S_{X}$ has at least one extreme point. The following statements are equivalent.

\footnotetext{
${ }^{1}$ Research supported by NSF Grant No. 25222.
} 
(1) For every two Banach spaces $Z \supset Y$ with $\operatorname{dim} Z=3, \operatorname{dim} Y=2$, every operator $T$ from $Y$ into $X$, and every $\epsilon>0$ there is an extension $\tilde{T}$ of $T$ from $Z$ into $X$ with $\|\tilde{T}\| \leqq(1+\epsilon)\|T\|$.

(2) For every two Banach spaces $Z \supset Y$, every compact operator $T$ from $Y$ into $X$, and every $\epsilon>0$ there is a compact extension $\tilde{T}$ of $T$ from $Z$ into $X$ with $\|\tilde{T}\| \leqq(1+\epsilon)\|T\|$.

(3) For every collection of four mutually intersecting cells in $X$ whose centers are in a two-dimensional subspace of $X$ there is a point common to all four cells.

For further properties equivalent to (1)-(3) and for functional representations of $X$ and its conjugates (e.g. $X$ satisfies (1)-(3) if and only if $X^{*}$ is an $L_{1}$ space) we refer the reader to [7] (cf. also [8, Theorem 1]). It seems likely that Theorem 1 holds also if $S_{X}$ has no extreme points (in [7] it is shown that if we replace 3 and 2 by 4 and 3 respectively, in the statement (1) above then it is equivalent to (2) even if $S_{X}$ has no extreme points). The essential step in the proof of Theorem 1 is to show that (3) implies that $X^{*}$ is an $L_{1}$ space. This is done by introducing an order in $X$ (as in [9] and [6]) and then showing, by using (3), that $X^{*}$ is (in the order induced by $X$ ) an abstract $L$ space in the sense of Kakutani [4].

The characterization of the spaces having stronger extension properties than (1) or (2), for example of spaces having the same properties but with $\epsilon=0$, presents many difficulties. However for the most important class of spaces satisfying (1)-(3), that is the $C(K)$ spaces (cf. [8, Theorem 3] for a characterization of these spaces among all the spaces satisfying (1)-(3)), the situation is simpler. We have

THEOREM 2. Let $X$ be the space $C(K)$ of all the continuous real-valued functions on the compact Hausdorff space $K$. The following statements are equivalent.

(a) For every two Banach spaces $Z \supset Y$ with $\operatorname{dim} Z=3, \operatorname{dim} Y=2$ and every operator $T$ from $Y$ into $X$ there is a norm preserving extension $\tilde{T}$ of $T$ from $Z$ into $X$.

(b) For every two Banach spaces $Z \supset Y$ with $\operatorname{dim} Z / Y=1$, and every operator $T$ from $Y$ into $X$ with a separable range there is a norm preserving extension $\tilde{T}$ of $T$ from $Z$ into $X$.

(c) $K$ is an $F$ space in the terminology of Gillman and Jerison [2], that is every two disjoint open $F_{\sigma}$ sets in $K$ have disjoint closures.

The equivalence of (b) and (c) is due, essentially, to Aronszajn and Panitchpakdi [1]. Properties (a) and (b) are not equivalent without the assumption that $X=C(K)$ (the space $c_{0}$ satisfies (a) but not (b)). If we assume the continuum hypothesis then it is not difficult to 
show that (for general Banach spaces $X$ ) property (b) is equivalent to the extension property obtained from it by discarding the requirement that $\operatorname{dim} Z / Y=1$. It is clear that in the extension properties in Theorems 1 and 2 it is sufficient to consider only operators $T$ which are isometries.

3. That finite dimensional spaces whose unit cell is a polyhedron are important in the study of norm preserving extension of operators, was pointed out already in [7] (cf. also [8, Theorem 2]). Here this question is studied further.

Theorem 3. Let $X$ be a Banach space such that $S_{X^{*}}$ is w* sequentially compact, and let $Y$ be a subspace of $X$. If for every $Z \supset Y$ with $\operatorname{dim} Z / Y$ $=1$ there is an operator with norm one from $Z$ into $X$ whose restriction to $Y$ is the identity then $Y$ is finite-dimensional and its unit cell is a polyhedron.

The requirement on $S_{X^{*}}$ appearing in Theorem 3 is satisfied, for example, if $X$ is separable or reflexive. It is easy to see that Theorem 3 does no longer hold if we discard the requirement on $S_{X^{*}}$. For example, take as $X$ a Banach space having an infinite dimensional subspace isometric to a $C(K)$ space with $K$ an $F$ space (cf. Theorem 2). The proof of Theorem 3 is based on the following

Lemma. Let $X$ be a Banach space. $X$ is finite-dimensional and its unit cell is a polyhedron if and only if there does not exist a sequence $\left\{x_{i}\right\}_{i=1}^{\infty}$ of points in $X$ satisfying $\left\|x_{i} \pm x_{j}\right\| \leqq\left\|x_{i}\right\|+\left\|x_{j}\right\|-1$ for every choice of signs and every $i \neq j$.

For finite-dimensional $X$ the assertion of the lemma is obvious. In the infinite-dimensional case the situation is not so simple because there exist infinite-dimensional Banach spaces all of whose finitedimensional subspaces have a polyhedron as unit cell.

Among the various applications of Theorem 3 we mention here only the following result which strengthens Theorem 2 of [8] in the separable case.

Theorem 4. Let $X$ be a separable Banach space. The following statements are equivalent.

(i) $X^{*}$ is an $L_{1}$ space and the unit cell of every finite-dimensional subspace of $X$ is a polyhedron.

(ii) For every two finite-dimensional Banach spaces $Z \supset Y$ with $\operatorname{dim} Z / Y=1$ and every operator $T$ from $Y$ into $X$ there is a norm preserving extension $\tilde{T}$ of $T$ from $Z$ into $X$. 
(iii) For every two Banach spaces $Z \supset Y$ and every operator $T$ from $Y$ into $X$ with a finite-dimensional range there is a norm preserving extension $\tilde{T}$ of $T$ from $Z$ into $X$ with $\widetilde{T}$ having also a finite dimensional range.

(i) and (iii) are equivalent even for nonseparable $X$ (cf. [8, Theorem 2]) but in general (ii) does not imply (iii) $(C(K)$ with $K$ an infinite-dimensional $F$ space satisfies (ii) but not (i)). We were not able to characterize the class of spaces having property (ii). It is clear from what was said above that this class includes the $C(K)$ spaces with $K$ an $F$ space and the (also nonseparable) spaces satisfying (i) of Theorem 4. Further it is clear that the class of spaces satisfying (ii) is closed under the operation of taking finite or infinite direct sums (in the $m$ norm). However not all the spaces satisfying (ii) can be obtained by taking direct sums of spaces of the two special types described above. We remark also that property (ii) can be formulated in terms of intersections of cells (cf. $[6 ; 7]$ ).

Another result concerning norm-preserving extension is

TheOREm 5. Let $X$ be a Banach space such that the extreme points in $X^{*}$ have a limiting point (i.e. are not isolated, the topology being the norm topology). Then there is a compact operator $T$ from $X$ into the space $c$ of convergent sequences and a space $Y \supset X$ with $\operatorname{dim} Y / X=1$ such that $T$ has no norm preserving extension from $Y$ into $c$.

Observe that $c$ (as any $C(K)$ space) satisfies (2) of Theorem 1. Theorem 5 can be easily reformulated as a result concerning the possibility of a continuous and norm preserving extension of a sequence of functionals (cf. the discussion in [7, Chapter 2]). The space $Y$ appearing in Theorem 5 is constructed by considering the dual problem of the continuity of the nearest point map from $Y^{*}$ to its one-dimensional subspace $X^{\perp}$. For finite-dimensional spaces $X$, also the converse of Theorem 5 holds and thus the possibility of extending, in a norm preserving manner, all operators from $X$ to $c$ characterizes the spaces $X$ with a polyhedron as unit cell among the finite-dimensional spaces (this characterization follows also from Theorem 3).

4. In some situations involving extension of operators (as in Theorem 3) separable and reflexive spaces behave in a similar manner. One reason for this is, perhaps, the following simple proposition.

Proposition. Let $X$ be a reflexive Banach space having the metric approximation property. Let $Y$ be a separable subspace of $X$. Then there is a separable $Z$ with $X \supset Z \supset Y$ such that there is a projection with norm 1 from $X$ onto $Z$. 
The notion of the metric approximation property (M.A.P.) was introduced by Grothendieck [3]. It is conjectured that all reflexive (and perhaps even all) Banach spaces have the M.A.P. A Banach space $X$ has the M.A.P. if for every compact $K \subset X$ and every $\epsilon>0$ there is an operator $T$ from $X$ into itself with a finite-dimensional range such that $\|T\|=1$ and $\|T x-x\| \leqq \epsilon$ for $x \in K$. The proof of the proposition is as follows. Let $\left\{y_{n}\right\}_{n=1}^{\infty}$ be a dense sequence in $Y$. By the M.A.P. we can choose, inductively, a sequence of operators $T_{n}$ from $X$ into itself and an increasing sequence of finite-dimensional subspaces $X_{n}$ of $X$ such that $\left\{y_{i}\right\}_{i=1}^{n} \subset X_{n}, T_{n} X \subset X_{n},\left\|T_{n}\right\|=1$, and $\left\|T_{n} x-x\right\| \leqq n^{-1}$ for $x \in S_{X_{n-1}}$. Put $Z=\mathrm{Cl}\left(\cup X_{n}\right)$. A projection from $X$ onto $Z$ is any limiting point (in the weak operator topology for operators from $X$ into $Z$ ) of the sequence $\left\{T_{n}\right\}_{n=1}^{\infty}$ (cf. the proof of the Lemma in [5]).

\section{REFERENCES}

1. N. Aronszajn and P. Panitchpakdi, Extension of uniformly continuous transformations and hyperconvex metric spaces, Pacific J. Math. 6 (1956), 405-411.

2. L. Gillman and M. Jerison, Rings of continuous functions, Van Nostrand, New York, 1960.

3. A. Grothendieck, Produits tensoriels topologiques et espaces nucleaires, Mem. Amer. Math. Soc. No. 16 (1955), 140 pp.

4. S. Kakutani, Concrete representation of abstract L-spaces and the mean ergodic theorem, Ann. of Math. (2) 42 (1941), 523-537.

5. J. Lindenstrauss, On some subspaces of $l^{1}$ and $c_{0}$, Bull. Res. Council Israel $10 \mathrm{~F}$ (1961), 74-80. 1962.

6. - Extension of compact operators. II, Technical note No. 31, Jerusalem, 1962.

7. - Extension of compact operators. III, Technical note No. 32, Jerusalem,

8. - On the extension property for compact operators, Bull. Amer. Math. Soc. 68 (1962), 484-487.

9. L. Nachbin, $A$ theorem of the Hahn Banach type for linear transformations, Trans. Amer. Math. Soc. 68 (1950), 28-46.

YALE UNIVERSITY 